\title{
THE OPTICAL DESIGN OF THE SPIN MANIPULATION SYSTEM FOR THE SLAC LINEAR COLLMER'
}

\author{
T. H. F1EGL'TH
}

Stanford Linear Arcelertor Center, Stanford Liniverstly, Stanford. Cahforma 94909

\section{ABSTRACT}

The opucal design of the bean transport lines between the SLAC Linac and the electron damping ring and the design of part of the Linac lattice itself will be modified to accomodate three superconducting solenoids for the purpose of manipulating the polarization of the electron bearn. In ordet to allow arbitrary orimtation of the polarization vector, this design will be capaof $\checkmark$ compensating the fietds of two independent solenoids for artitrary strength ranging to $7.0 \mathrm{~T}$-m. The method of dealing with the corpling if the hetatron functions and the method of iandiung both the electron and posstron beams in the common region are discussed.

\section{INTRODUCtIoN}

fi has long been planned 1.2 .3 to collide a polarized electron beam with a positron beam at the Stanford Linear Collidet (SLC) to study the electroweak interactious. ${ }^{4.5}$ To provide a polarized bean, it will be necessary to produce a low energf: beam of polarized elect tons, shrink its transverse emittance in a damping ring. accelerate it to a bigh energy and transport it to the collision point. This process is illustrated in Fig. L. which shows the orientalion of the spin polarization vector at various regions of SLC. Fote that the beam is longitudinally polarized only in the region near the source and at the collision point itself To achipve this of any olber desired orientation for colli. sions, the precession of this vectar in the magnetic guide fields must be controlled and compensated. At the electron damping rtag. the polarization rector is rotated into the vertical direction before :njection into the ring to avoid the depolarization caused by the energy spread of the beam and the energy dependence of the precession.

In the linat downstream of the damping ring, the polariza ton vector can be oriented such that it will became longitudinal (or have any other chosen orientation) at tbe collision point af. ter precessing many time through the SLC Arcs. In bes been show ${ }^{6}$ that compensation for the energ dependence and the vertical defiection of the Arc guide field will require the capa. bility of providing an almost arbitmery orientation of the spin polaritation vectof in the linac. This orientation will change as the center-of.mass etergy of the bearns al the collision point is modified for particle physics experiments.

Here we describe bow solecoidal and dipole fields are ati. lized to manipulale the polarization vector it the region of the damping ring and bow the optical effects are compensaled.

\section{SPIN PRECESSION}

The precession motion for the magnetic moment of an eccelerating relativistic particle is siven by the solution of the Thoms-BMT equation, which is described in Ref. (7). From this veclor equation is oblained the expression for the magnitude of the precession angle abput the direction of the magnetic iuduction rector $\mathbf{B}$. which is either perpendicular to the particle velocity (as in a horizontal or vertical bending magnet) or par. allel to its velocity (as in the solenoids we iatead to use). For the bend mognets the piecession angle ti in the laboratory frome is given by.

$$
\text { viread }=(1+\nu) \theta_{\text {bend }} \text {. }
$$

where $\theta_{\text {had }}$ is the bending angle of the beam. and the "apin tuae $\bar{\nu}$ is given by

$$
\nu=\left(\frac{g-2}{2}\right) \frac{E}{m_{\mathrm{r}} c^{3}}=\frac{E(G e l) !}{0.49067}
$$

Hete $E$ is the beam energy and ( $\frac{y^{2}}{j}$ ! and $m$, are the anoma. lous magnetir moment and the mass of the elertron, tespectively. Thork supported tis the Department of Enetgy. contract DE-ACO3765500515
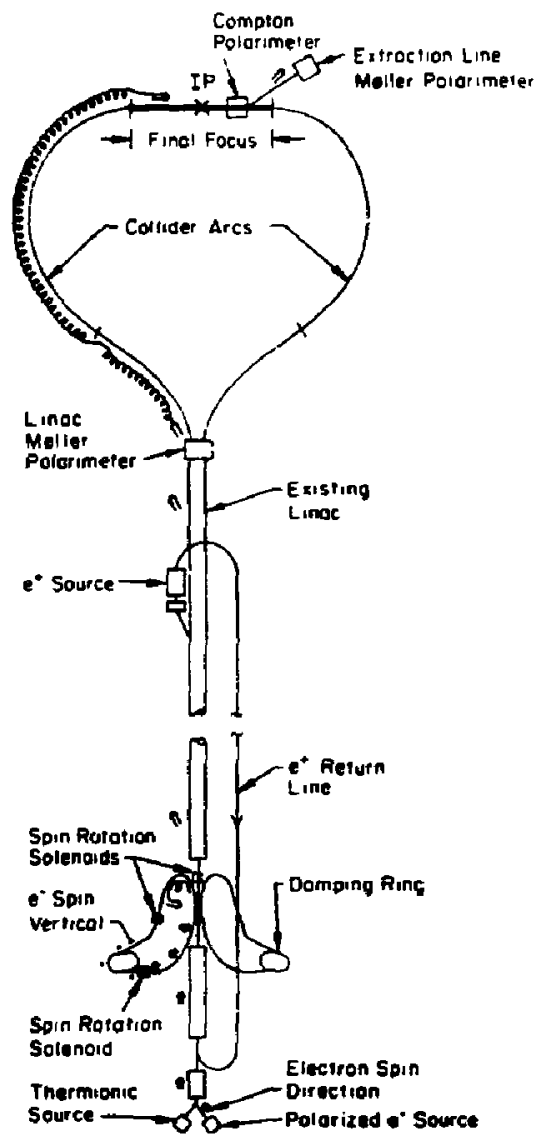

POLARIZATION IN TME OVERALL SLC LAYOUT

$10 \cdot 06$

Fig. J. Sthe matic fayout of SLC.

At the design enetgy of the damping riog of $1.21 \mathrm{GeV}, \nu=2 . \mathrm{if}$. Thus, when the bests is bent by $32.8^{\circ}$. the polarization vector will precess by $122.8^{\circ}$ in the laboratory, or $90^{\circ}$ with respect to the local velocity vector. The laboratory precession engle for the polarization vector of a beam passing shrough a constant solenozd field of length $L$ and flux density $B$ is given by

$$
\text { Uolemond }=\left[1+\left(\frac{g-2}{2}\right)\right] \frac{B L}{B_{\Delta \rho}} \text {. }
$$

where $B_{\theta} \rho$ is the usual magnetir rigidity of a besm of momentum $P$ given by $B_{0} p(T-m)=3.3756 P(G-V / C)$. Note thin $\left(\frac{2-2}{2}\right)=1.1596 \times 10^{-1}$ for electrons and can be neglected here. Foi a momentum $P$ equal to $1.21 \mathrm{GeV} / \mathrm{r}, B_{a p} \approx \uparrow \mathrm{T}-\mathrm{m}$. For a precession angle v'role rigend $=\pi / 2$. E4. 3 gives a required solenoid strength of $B L=6: 3 \mathrm{~T} \cdot \mathrm{m}$. At the damping ring three solenoids. each rapable of allaning this strength. wili be used for spin 
polarization nanipulation. These are superconducting magnets with a peak field of $4.2 \mathrm{~T}$ and an effective length of $1.65 \mathrm{~m}$.

\section{OPTICAL EFFECTS}

The optical effects of a solenoid are represented by a

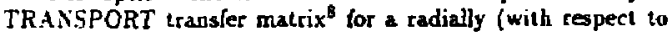
the bean axis) focusing element followed by a roll about the beam axis of the curvilinear coordinate system moving with the beam. The azimuthally symmetric focusiog is independent of the charge of the particles or the reversal of the direction of the solenoidal field. Tbe radial focal length $f$ is given by

$$
\frac{1}{f}=\frac{B}{2 B_{\circ p}} \sin \frac{B L}{2 B_{o p}} \quad \text { (meters). }
$$

The direction of the roli angle about the beam axis does depend upon the particle charge and the field direction. Its magnitude is given by

$$
\theta_{\text {roll }}=\frac{B L}{2 B_{\Delta} \rho}=\frac{1}{2} \psi_{\text {rolmold }}
$$

For the superconducting calenoids operating \& $6.3 \mathrm{~T} \cdot \mathrm{m}$ the tadial fucal length is $3.0 \mathrm{~m}$ and the roll angle about the beam axis is $45^{\circ}$ which is, of course, ball of the precession angle. These are large optical effects which must be compensated.

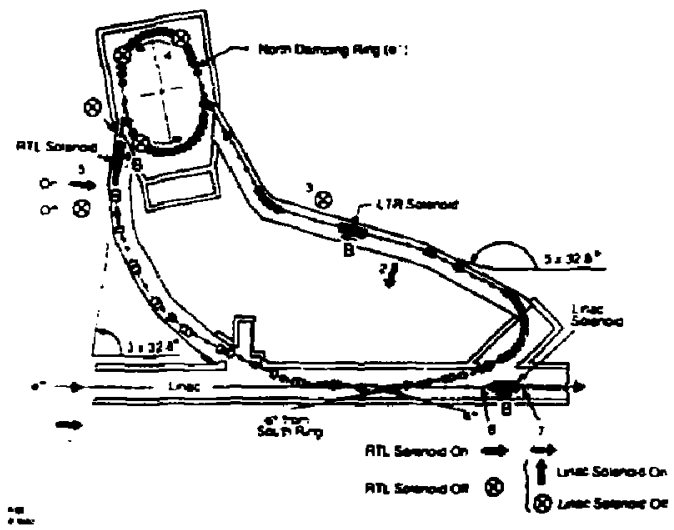

Fig a. Polartiation mantpulation al electon damping ring.

\section{SPIN MaNIPULATJON}

Figure 2 shows a plan view of the SLC electros dampiag tung ac. $I$ its two transport lines. At position (1) the beam is longitudinally polarized. The beam is extracted from the linac into the Linar.to-Riug (LTR) beam lipe and best through an angle of $164^{\circ}\left(5 \times 32.8^{\circ}\right)$ to position (2) whese the polarization vertor has precessed about the vertical axis by $5 \times 90^{\circ}$ and is now perpendicular to the benm velocity. It then precessed in the LTR solenoid by $90^{\circ}$ to the vertical dirextion at (3). This orientation is preserved in the damping riog (4) and wben the beam is extracted (5) the polarization vector precesses in the Aing-to-Limac (KTL) wolenoid by angle which can have any value between zero and $\pm 90^{\circ}$. The resultiag vector then precesses about the vertical exis by $3 \times 90^{\circ}$ white the beam is being bent by $98.4^{\circ}\left(3 \times 32.8^{\circ}\right)$ while returning te the linac end its solenoid at (6). Here the vector is rotated to its final orientation by precessing in the linac solenoid through an angle up to $\pm 90^{\circ}$. Independent control of the precession in the RTL solenoid and the linac solenoid allow ap arbitrary orientation of the polarizen. tion vector of the beam injected into the linac.

The optical ce rpensation for the LTR solenoid is simple berause it has but two stales (polarity change does not affect forusing). It is either at its full strength of $6.3 \mathrm{~T} \cdot \mathrm{m}$ to precess the spin by $\pm 90^{\circ}$ or it is off. On the other hand, the compensa. ton for the RTL solenoid and for the linac solenoid must work for all possible combinations of polarities and field strengths up

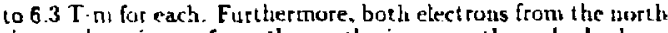
ring and positrons from the south ring pass through the linar solenoid and so their respective coprdinates undergo a roll in opposite directions aboul the beam axis. The placement of this solenoid in the linac (where there is no dispersion) is neressary to avoid the uncorrectable vertical dispersion which would resuli if the solenoid were placed in the RTL hine where the horizontal dispersion is purposely large for bunch compression

\section{ROUND BEAMS AND}

\section{ChARGE INDEPENDENT OPTICS}

The second-order differential equations satisfied by the hori

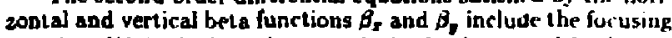
function $f(s)$ wbich is the sarne in both planes and both par ticle charges for a drifh $(k=0)$ or for a solenoid $\left(k_{s}=k_{y}\right.$ ) Thus, if the ibitial conditions are the same. $\phi_{x}=\beta_{r}$ and $y_{r}=S_{v}$ (prime indicates differentiation with respert to path leigth ) the colutions are also the same and $\theta_{2}$ equals $\theta_{1}$ everywhere in the solenoid and adjacent drifts. This azimutbal symmetry of the optical functions simplifies matching to the initial conditiuns te guired for these functions in the following downitrean system. The matching problem associated with varying rolls is remorrd and bence the dependence of the sign of the roll on the charge of the particles also becomes unimportant. This reduces thr optical problem to that of obtaining solutions wherein only the varying focusing of a solenoid is compensated in ordet to kepp constant values for $\theta_{2}, \beta_{1}, B_{4}^{\prime \prime}$ and $\xi_{4}$ as input to the downstream system.

If the optical functions in the two planes are everywhere equal and the transverse emittances are also equal $\left(c_{z}=c_{y}=e\right)$ then the transwerse beam sizes will be equal. $\sigma_{a}=\sigma_{z}=\sqrt{d t}$ and the beam is round. Such equal transverse emittanecs have been assumed for the SLC design and have been ohtained by operating the damping rings in a fully coupled mode where the horizontal and vertical fractional tunes are equal. !! is important to note that while making the beta functions equal it hot h planes belps to simplify the mat ching of these functions thes dues not eliminate the inherent cross plane coupling in the tronsfer matrices cowsed by the solenouds. This becomes readily apparent with flat beans(initially unequal emitlances).

\section{LTR SOLENOID OPTICS}

In Fig. 3 the beta functions for the LTR beam line are plot. ted. The solenoid is placed in a dispersion free region midway be. tween two points in the upstream and doninstream lattice where the values of the beta functions are the same. Quadrupoles are placed to be mirror symmetric about the center of the solenoid and their field strengths adjusted, also symmetrically, so that - double waist $\left(\beta_{g}^{\prime}=\beta_{7}^{\prime}=0\right)$ occurs at the solenoid center. The resulting beta functions now have the sarne mirror symmetry and match to both the upstream and downstron! lattices. For the case where the solenoid is turned on [see Fig. $3(\mathrm{a})$ a solution is obtained with the edditional const/aint that the incoming beta functions be matched to a "round" wast $\left(\beta_{2}=\theta_{y}, \quad G_{z}=\theta_{y}=0\right)$ at the center of the solessoid. When the solenoid is turned of [soe Fig. $3(b))$, the double waist requirement is sufficient to ensure a downstream match. By not requiring aximuthal symmetry for both cuses, the number of quadrupoles needed is reduced.

\section{RTL AND LINAC SOLENOIDS}

The purpose and design of the RIL beam line is described in Ref. 3. It can be divided into two regions. The fist, thr mateheng regnon lies between the damping ring and the sacond, or compression and correction region. The matching region is dispersion free and its main components are four quadrupolis. the spin precessing solenoid and an RF accelerating wavrguidt", This last structure is part of the bunch compression system in RTL and does not affect the optics discussed here. The compres. sion and correction region which follows has been corrected for chromatirs aberrations up to serond-order and is thereby well represented using only its first order transfer malrix which is equal to the tdentity. The solenoid and compensating optics 


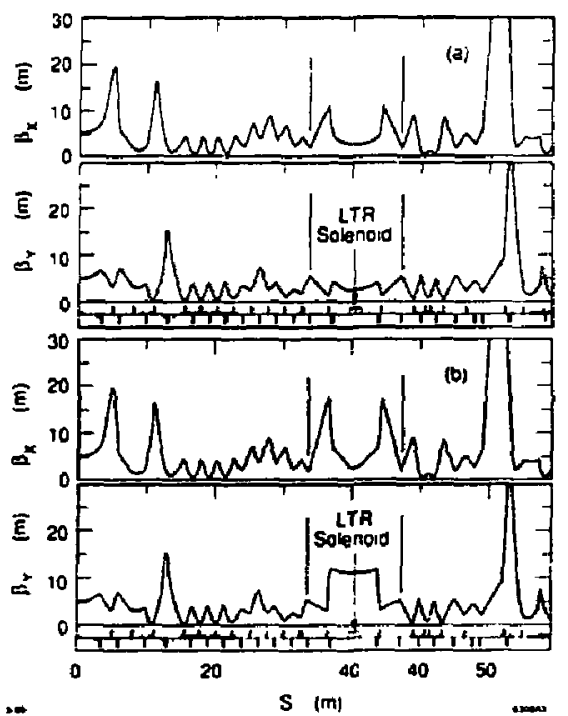

Fig. 9. Beta fanctions in LTR wath fal solenand field strength at $6.9 T$-m and $(b)$ off.

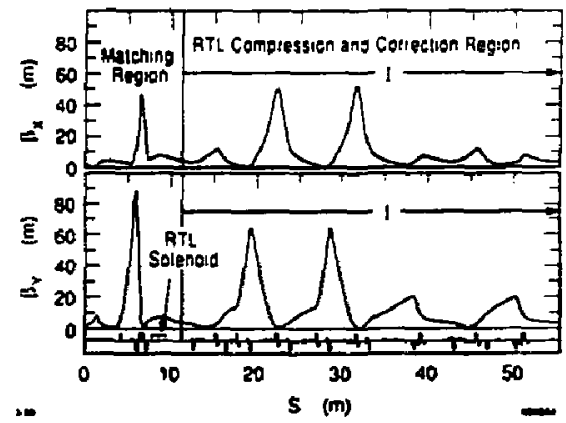

Fig. 4. Beta funetions in RTL metehing region and compression and correction repion.

do not affect its function or its second-order optics. The matching regions in both the electron and the positron return lines are the anme except that there in no cotenoid in the positron lipe. With the solenoid in the electron return line turued of the strengths of the four matching quedrupoles in euch are set to the same reapective values so that the two beams are matched to the same conditions at the entraces to their respective compression and correction region. Then, because this latter region cas be represented by the identity transformation these beam parameters are reproduced to the entrance to the linac. This is illustrated for the electron bean in Fig. 4, where the position of the four matching quadrupoles and the RTL solenoid are indicated. The peaks where $\beta_{z}$ and $\beta_{y}$ reach values near $60 \mathrm{~m}$ within the RTL beamline occur because of an unavoid. able mismatch between the two regions. The input values have been adjusted to minimize there peaks. When the field of the solenoid in the electron ben line is excited, the effect of its focusing is cuinpensated by the four upitream quadrupoles while those it $h_{1}$ - position beam line are not changed. Both bears remain ideutical entering the linac where a third set of four linae quadrupoles common now to both besms is used to compensate the focusing of the linar solenoid. In these four quadrupoles as

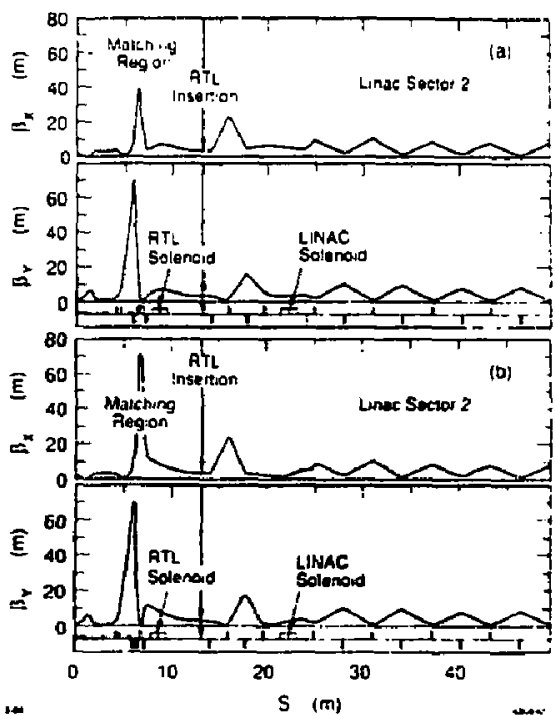

Fig. 5. Beto functions in RTL and inac solenosdo when the idertify part of RTI. is remoied: (a) RTL solenoud on. linoc sole noid off. (b) RTL solenoid off. linar sale noid on

in the linac downstrean, the beta functions for the electrons $(-)$ and positions $(+)$ are related by a $90^{\circ}$ roll about the beam axis, t.e., $\sigma_{x}\left(-1=\beta_{y}(+), S_{z}(-)=\xi_{y}(+), S_{1}(-)=\beta_{x}(+)\right.$ and $A_{y}(-)=B_{z}^{\prime}(+)$. For a quadrupole. $90^{\circ}$ roll is equivalent to a polarity reversal hence this relationship once established wil! remain crue in a sequence of quadrupoles and drifs. The az. imuthally symmetric functions used here for input are but a special case where this relationship is true. In Fig. 5 the part of the RTL which is represented by the identity matrix is removed so the optical relationship of the solenoids and their respective compensating quadrupoles cas be clearly seen. Two represers. tative values for the solenoids are chosen for illustration but all values are attainable. The functions for the positron beam are obtained by wetting the strength of the first solenoid to zero and interchanging the horizontal and vertical planes $\left(90^{\circ}\right.$ roll). The upatream compensation leaves the beams exiting the linar colenoid unchanged so the quadrupoles downstream in the linar also remuin the constant excitation.

\section{CONCLUSION}

An optics for compensating the efiects the SLC spin manipulating wolenoids bas been designed in which the bet in functions for botb electrons and pasitrons can remain properly makched. If these beams have equal transverse emittances the transverse coupling will not affect the observed spot sizes.

\section{REPERENCES}

1. SLC Denigo Bendbook, SLAC. December 1984

2. R. H Stiening. SLAC Report AATF/80/28, August 1980

3. T. I Fieguth and J J. Murray, Destyn of the SLC Damping Ring to Liner Theseport $L$ ines, I2th Int. Conf on High Energy Accel., Fermilab, pp. 401-403, (1983)

4. C. C. Prescolt. Prot Int Symp. Bigh Energy Piytits with Polurised Beams and Polariced Targets (Lausmnne 1980), ed C Joseph and J Soffer (Biskhauser Verlag. Basel, 1981)

5. D Blockus et al. Proposal for Polariation at the SLC. SI.AC Proposal SLC Upgrade o! April 1986

6. J J Murray. SLC Polarization Group internal Memarandum (1986)

i Bryan W Monlague Phps Rep. 113, No 1. 8-13 (1984)

B. K L Brown at al, CERN 80-04, Mareb 1980 


\section{DISCLAIMER}

This repon was prepared as an acount of wort sponsored by ab agency of the United States Government. Neither the United States Government not any agency thereof, nor any of their employes, mates any warnaty. express or implied, of assumes any legal liability or responssbility fot the accuracy, completeness, or usefulness of any information, apparatus. product, or process diselosed. or represents that is use would not infringe privately owned rithts. Reter. ence hercin to any specifte commercial product. process, or servioe by trade name. trademark. manulacturer. or otherwise does not necessarily constitute or imply its endorsement, recommendation, or favaring by the United States Government or any agency thereol The views and opinions of authors expresed herein do not necescarily stale of renext those of the Uniled States Government or any agency thereof. 